\title{
Concerning the chiral phase transition in QED
}

\author{
J. C. Montero \\ Laboratoire de Physique Mathématique, Université des Sciences et Techniques du Languedoc, \\ 34095 Montpellier CEDEX 5, France \\ V. Pleitez \\ Instituto de Física Teórica, Universidade Estadual Paulista, Rua Pamplona 145 CEP 01405 \\ São Paulo, São Paulo, Brazil \\ (Received 26 March 1990)
}

\begin{abstract}
Using the effective potential for composite operators but without using a variational approach, we show the possible existence of a dynamical phase transition from a massive phase to a massless one.
\end{abstract}

The problem of the spontaneous breakdown of chiral symmetry with a composite operator in nonsoluble theories such as four-dimensional QED is an old and open one. ${ }^{1,2}$ Some time ago we showed, ${ }^{3}$ using the formalism of the effective potential for composite operators ${ }^{4}$ (EPCO), that in the ladder approximation, in quantum electrodynamics in a continuum space-time with a massless fermion, the latter gets a mass for every nonzero coupling constant. In this case the Goldstone theorem is evaded. ${ }^{5}$ The picture that arises, taking our results seriously, is in close analogy with the BCS theory of superconductivity in which any charge different from zero produces a condensate if not thermally disrupted. In Ref. 3 we calculated the EPCO using a variational approach and two asymptotic solutions for the Schwinger-Dyson (SD) equation for the fermion propagator (see below) which are valid for $0<\alpha=e^{2} / 4 \pi<\pi / 3$; that is, we are able to search for a critical coupling constant in the above interval for $\alpha$.

On the other hand, it is possible to consider the mechanism of spontaneous chiral-symmetry breaking in QED based on the analogy between this phenomenon and that of the "fall" of a particle to the center. 6 In this formalism the critical coupling constant is $\alpha_{c}=\pi / 3$, which separates the massless $\left(\alpha<\alpha_{c}\right)$ and the massive $\left(\alpha>\alpha_{c}\right)$ phases.

A third approach to the problem of chiral phase transition is the lattice technique. In noncompact lattice QED in the quenched approximation the critical coupling constant $\alpha_{c}=3.75$ has been found. ${ }^{7}$ However, using an improved action (having the same infrared behavior but differing in the ultraviolet part) the critical constant becomes $\alpha_{c} \simeq 2.00$. The authors of Ref. 7 have interpreted these results as an indication that the critical coupling constant is nonuniversal and is strongly influenced by the short-distance behavior of the gauge field theory. When fermions are introduced in the theory the phase transition survives. ${ }^{8}$ But in Ref. 8 fermions have a relatively large bare mass: $m=0.25$ and 0.20 in lattice units. Recently a study of the chiral phase transition in compact QED with light fermions $(m=0.1,0.2)$ gave a critical coupling constant lower than the previous ones: ${ }^{9}$ $\alpha_{c}=1.119$ for $m=0.10$ and $\alpha_{c}=1.088$ for $m=0.25$. In Ref. 9, the existence of a first-order transition for $m=0.10$ is also suggested.

Recently a lot of new and interesting results have been obtained in noncompact QED. For example, with four species of light fermion (i.e., $N_{f}=4$ ) and bare fermion mass $m=0.0125$ in lattice units simulation data confirm that there is a second-order phase transition to a strongcoupling phase where chiral symmetry is broken. ${ }^{10}$ There is also a finite-size effect which is a nontrivial effect which can be interpreted as an indication that the phase transition is associated with an ultraviolet-stable fixed point of an interaction theory of meson bound states. The theory also shows a real temperature and flavor number dependence. $^{11}$

We can think that our results using EPCO with a variational approach which gives us $\alpha_{c}=0$ (Ref. 3) could be consistent with the collapse picture ${ }^{6}$ and lattice technique $^{7-11}$ in which $\alpha_{c} \gtrsim 1$ if there is a phase transition with a critical coupling constant $\alpha_{c}^{\prime}$, with $0<\alpha_{c}^{\prime}<1$ but now the transition being from a massive phase $\left(\alpha<\alpha_{c}^{\prime}\right)$ to a massless one $\left(\alpha>\alpha_{c}^{\prime}\right)$. Then the other phase transition with $\alpha_{c} \gtrsim 1$ from a massless phase to a massive one could occur. If this is true the reason we did not find the critical coupling constant $\alpha_{c}^{\prime}$ in Ref. 3 could be an artifact of the variational approach or of the two-loop effective potential. On the other hand, in order to have physically significant results we must use in the effective potential exact solutions of the SD equation or a variational approach. Of course, exact solutions to the nonlinear SD equation are not known; for this reason the variational approach is the preferred one in the literature for both Abelian $^{3,4,12}$ and non-Abelian ${ }^{13}$ theories.

In this paper we want to point out that in the case of QED in the latter approximation and with $-p^{2} \rightarrow \infty$ we have asymptotic solutions and for this reason we can use them with some confidence in the effective potential without using a variational approach. This could not be done in QCD in which even asymptotic solutions are only approximates. ${ }^{13,14}$

We shall use the usual situation ${ }^{3,4,13}$ of the free vertex and 


$$
\begin{aligned}
& S(P)=\frac{i}{\not p}, \quad G^{-1}(p)=S^{-1}(p)-\Sigma(p), \\
& D^{\mu v}(p)=\frac{-i}{p^{2}}\left[g^{\mu v}-\frac{p^{\mu} p^{v}}{p^{2}}\right),
\end{aligned}
$$

with $\Sigma(p)$ being a nontrivial solution of the SD equation for the fermion propagator which in this approximation becomes

$$
i \Sigma(p)=3 e^{2} \int \frac{d^{4} k}{(2 \pi)^{4}} \frac{\Sigma(k)}{(p-k)^{2}\left[k^{2}-\Sigma^{2}(k)\right]} .
$$

After writing the last equation in Euclidean space and integrating in the angular variables we can obtain in a straightforward way the SD equation in the differential form

$$
\frac{d^{2} \Sigma(p)}{d p^{2}}+\frac{3}{p} \frac{d \Sigma(p)}{d p}+\frac{4 \epsilon \Sigma(p)}{p^{2}+\Sigma^{2}(p)}=0 .
$$

With appropriate boundary conditions, (2) and (3) are equivalent. Both Eqs. (2) and (3) are nonlinear and no analytical solutions have been found. However, if we consider the regime in which $p^{2} \gg \Sigma^{2}(p)$ we can write Eq. (3) as

$$
\frac{d^{2} \Sigma(p)}{d p^{2}}+\frac{3}{p} \frac{d \Sigma(p)}{d p}+4 \epsilon \frac{\Sigma(p)}{p^{2}}=0,
$$

and it is easy to show that this linearized SD equation has exact solutions of the form

$$
\Sigma(p) \propto f(p), \quad f(p)=\left(\frac{p^{2}}{\mu^{2}}\right)^{-\gamma},
$$

since $\gamma(1-\gamma)=\epsilon$. That is, $\gamma_{ \pm}=\frac{1}{2}\left[1 \pm(1-4 \epsilon)^{1 / 2}\right]$ and $\epsilon=3 a / 4 \pi$. is

The Euclidean effective potential at the two-loop level

$$
\begin{array}{r}
V(\Sigma)=2 \int \frac{d^{4} p}{(2 \pi)^{4}}\left[\ln \left[1+\frac{\Sigma^{2}(p)}{p^{2}}\right]\right. \\
\left.-\frac{2 \Sigma^{2}(p)}{p^{2}+\Sigma^{2}(p)}\right]+V_{2}(\Sigma),
\end{array}
$$

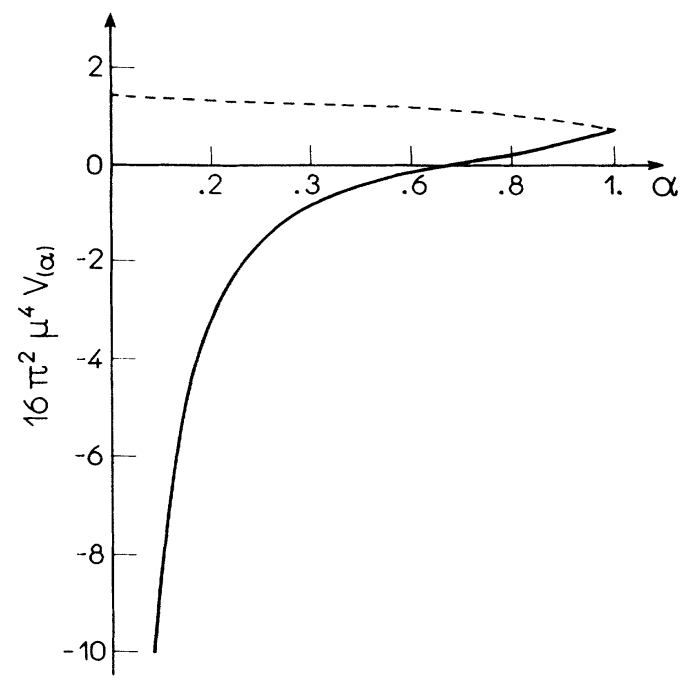

FIG. 1. The effective potential as a function of the coupling constant. With the $f_{-}(p)$ solution (solid curve) there is a critical coupling constant $\left(\alpha_{c}=0.67\right)$; this is not the case for the $f_{+}(p)$ solution (dashed curve).

with

$$
\begin{aligned}
V_{2}(\Sigma)=-6 e^{2} \int & \frac{d^{4} p}{(2 \pi)^{4}} \frac{\Sigma(p)}{p^{2}+\Sigma^{2}(p)} \\
& \times \int \frac{d^{4} k}{(2 \pi)^{4}} \frac{\Sigma(k)}{(p-k)^{2}\left[k^{2}+\Sigma^{2}(k)\right]} .
\end{aligned}
$$

In this work we use the ansat $z^{3,13}$

$$
\Sigma_{ \pm}(p)=\mu\left[\theta(\mu-p)+f_{ \pm}(p) \theta(p-\mu)\right]
$$

with $f_{ \pm}(p)$ given in Eq. (5) with $\gamma=\gamma_{ \pm}$. Using expression (8) in (6) with $x=p^{2} / \mu^{2}$ and $y=k^{2} / \mu^{2}$ we obtain

$$
V(\mu, \epsilon)=\frac{\mu^{4}}{16 \pi^{2}}\left\{3-4 \ln 2-2 \int_{1}^{\infty} d x x\left[\ln \left(1+\frac{f^{2}(x)}{x}\right)-\frac{2 f^{2}(x)}{x+f^{2}(x)}\right]+V_{2}(\epsilon)\right\},
$$

where

$$
\begin{aligned}
V_{2}(\epsilon)=-4 \epsilon[1 & -\ln 2-\frac{1}{2} \ln ^{2} 2+(1-\ln 2) \int_{1}^{\infty} \frac{d x}{x} \frac{f(x)}{x+f^{2}(x)} \\
& \left.+\frac{1}{2}\left[\int_{1}^{\infty} \frac{d x}{x} \frac{f(x)}{x+f^{2}(x)} \int_{1}^{x} d y \frac{f(y)}{y+f^{2}(y)}+\int_{1}^{\infty} d x \frac{f(x)}{x+f^{2}(x)} \int_{x}^{\infty} \frac{d y}{y} \frac{f(y)}{y+f^{2}(y)}\right]\right] .
\end{aligned}
$$

The above expressions (9) and (10) were calculated numerically as a function of $\epsilon$ (or $\alpha$ ). We can search for a possible phase transition, looking for a change of sign in the effective potential, that is, if a critical coupling con- stant exists such that below it $\left(\alpha<\alpha_{c}\right)$ the potential is negative. This is indeed the case for the irregular solution $\gamma=\gamma_{-}$. The results are shown in Fig. 1. For the $f_{-}(p)$ solution (solid curve) the critical coupling con- 
stant is $\alpha_{c}=0.67$. For the $f_{+}(p)$ solution (dashed curve) there is no change of sign. Then for $\alpha>\alpha_{c}=0.67$ the theory has massless fermions and then the second phase transition should appear with a stronger critical coupling constant $\alpha_{c} \simeq \pi / 3$.

We are well aware that our results do not prove the existence of this phase transition. We only point out the possibility of this. The effective potential in the massive phase is unbounded below. This is because we are assuming the existence of an eigenvalue condition; that is, the $\beta$ function in the renormalization-group equations vanishes ${ }^{15}$ and in this case the effective potential is a simple power. ${ }^{2}$ But this is exactly why we think that the change of sign of the effective potential is a nontrivial result. Us- ing a variational approach it is possible to get an effective potential which is bounded below, but in this case no transition to a massless phase appears.

Only by obtaining better solutions to the SD equations or through agreement with other techniques can we confirm if the possibility we have pointed out in this paper is realized in nature or not.

One of us (J.C.M.) thanks the Conselho de Desenvolvimento Científico e Technológico (CNPq) for financial support and Laboratoire de Physique Mathématique, Universite des Sciences et Techniques du Languedoc-Montpellier, for their kind hospitality.
${ }^{1}$ G. S. Guralnik, C. R. Hagen, and T. W. B. Kibble, in Advances in Particle Physics, edited by R. L. Cool and R. E. Marshak (Interscience, New York, 1968), Vol. 2.

${ }^{2}$ S. Coleman and E. Weinberg, Phys. Rev. D 7, 1888 (1973).

${ }^{3}$ J. C. Montero and V. Pleitez, Phys. Rev. D 35, 2579 (1987).

${ }^{4}$ J. Cornwall, R. Jackiw, and E. Tomboulis, Phys. Rev. D 10, 2428 (1974).

${ }^{5}$ Th. Maris and G. Jacob, Phys. Rev. Lett. 17, 1300 (1966); H. Pagels, ibid. 28, 1482 (1972); Phys. Rev. D 7, 3689 (1973).

${ }^{6}$ P. Fomin, V. Gusynin, V. Miransky, and Yu. Sitenco, Riv. Nuovo Cimento 6, 1 (1983); V. Miransky, Nuovo Cimento 90A, 149 (1985); Yad. Fiz. 43, 482 (1986) [Sov. J. Nucl. Phys. 43, 307 (1986)].

${ }^{7}$ J. Bartolomew, J. Kogut, S. Shenker, J. Sloan, M. Stone, H. Wyld, J. Shigemitsu, and D. Sinclair, Nucl. Phys. B230
[FS10], 222 (1984).

${ }^{8}$ V. Azcoiti, A. Cruz, E. Dagotto, A. Moreo, and A. Lugo, Phys. Lett. B 175, 202 (1986).

${ }^{9}$ J. B. Kogut and E. Dagotto, Phys. Rev. Lett. 59, 617 (1987).

${ }^{10}$ J. B. Kogut, E. Dagotto, and A. Kocic, Phys. Rev. Lett. 60, 772 (1988).

${ }^{11}$ E. Dagotto, A. Kocic, and J. B. Kogut, Phys. Lett. B 232, 235 (1989).

${ }^{12}$ V. P. Gusynin and V. A. Miransky, Yad. Fiz. 31, 787 (1980) [Sov. J. Nucl. Phys. 31, 407 (1980)].

${ }^{13}$ P. Castorina and S.-Y. Pi, Phys. Rev. D 31, 411 (1985); V. P. Gusynin and Yu. A. Sitenko, Z. Phys. C 29, 547 (1985).

${ }^{14}$ J. C. Montero and V. Pleitez, Z. Phys. C 42, 271 (1989).

${ }^{15}$ M. Baker, K. Johnson, and R. Willey, Phys. Rev. 136, B1111 (1964). 